\title{
Passenger car vs. truck tires - different development priorities
}

Stephan Brückner, Hankook Tire Europe GmbH

This manuscript is not available according to publishing restriction.

Thank you for your understanding. 